\title{
An Analysis of the Effects of Population Structure on Scalable Multiobjective Optimization Problems
}

\author{
Michael Kirley \\ Department of Computer Science \& Software \\ Engineering \\ The University of Melbourne \\ Victoria, Australia \\ mkirley@csse.unimelb.edu.au
}

\author{
Robert Stewart \\ Department of Computer Science \& Software \\ Engineering \\ The University of Melbourne \\ Victoria, Australia \\ robertls@csse.unimelb.edu.au
}

\begin{abstract}
Multiobjective evolutionary algorithms (MOEA) are an effective tool for solving search and optimization problems containing several incommensurable and possibly conflicting objectives. Unfortunately, many MOEAs face difficulties in solving problems when the number of objectives increases. In this paper, we investigate the efficacy of spatially structured MOEAs for scalable multiobjective problems. The algorithm is an extension of the standard cellular evolutionary algorithm, where the population is mapped to nodes of alternative complex networks. A selection regime based on a non-dominance rating and a crowding mechanism guides the evolutionary trajectory and an $\epsilon$-dominance external archive is used to maintain a spread of solutions across the Paretooptimal front. An important outcome of this work is the classification of the network models based on their impact on convergence speed and solution quality as the number of objectives increases for a given problem.
\end{abstract}

\section{Categories and Subject Descriptors}

G.1 [Numerical analysis]: Optimization; I.2 [Artificial Intelligence]: Problem Solving, Control Methods, and Search

\section{General Terms}

Algorithms, Performance, Experimentation

\section{Keywords}

Complex network, Parallel evolutionary algorithm, Multiobjective optimization

\section{INTRODUCTION}

Multiobjective evolutionary algorithms (MOEA) are now an established technique used to find a set of Pareto-optimal solutions when confronted with search and optimization problems containing several incommensurable and possibly

Permission to make digital or hard copies of all or part of this work for personal or classroom use is granted without fee provided that copies are not made or distributed for profit or commercial advantage and that copies bear this notice and the full citation on the first page. To copy otherwise, to republish, to post on servers or to redistribute to lists, requires prior specific permission and/or a fee.

GECCO'07, July 7-11, 2007, London, England, United Kingdom.

Copyright 2007 ACM 978-1-59593-697-4/07/0007 ...\$5.00. conflicting objectives [3, 4]. Well known MOEAs include NSGA-II [6], SPEA2 [18] and PAES [14].

Unfortunately, many MOEAs face difficulites in solving problems when the number of objectives increases. This may be attributed to the fact that the dimensionality of the objective space has increased and changes in the interaction patterns between decision variables may cause a large proportion of a random initial population to be non-dominated with respect to each other [4]. Deb and Sundra [7] suggest that a number of factors contribute to performance degradation. These include the problems associated with visualising higher-dimensional objective space, the trade-off between exploration/exploitation of the search space as a consequence of reduced selection pressure in small populations, and, the exponentially larger number of points required to represent a higher-dimensional Pareto-optimal front.

A number of papers have appeared examining the role that spatial structure plays in guiding evolutionary processes in an evolutionary computation context in the last two years. Notable examples include the works of Alba and Dorronsoro [1], Giacobini et. al., [10, 11] and Bryden et. al., [2]. The results reported, using single objective problems, show how the topology of the network (or graph) used to constrain the interactions between individuals has a direct impact on the overall behaviour of the evolving population. Bryden et. al., suggest that the graph yielding superior performance is problem dependent. Giacobini et. al., highlight significant differences between the so called "small-world" models [16] and regular lattices.

An interesting question, therefore, is how does the underlying spatial structure of an evolving population impact on the quality of solutions found for multiobjectiove optimization problems? Recently, a complex network-based MOEA was introduced [13], which combined some of the ideas outlined above with a Pareto-based algorithm for multiobjective problems. Fundamentally, the model was a diffusionbased (or cellular) evolutionary algorithm where the regular lattice was replaced by a small-world, scale-free or random network [9]. An important feature of the algorithm was the variation in connectivity (node degree) and corresponding selection pressure across a given network. Reported results suggest that there were signficant differences between the network architectures considered using the well-known ZDT benchmark multiobjective problems [19].

In this paper, we use the complex network-based MOEA as a framework for analyzing the effects of population structure on scalable multiobjective optimization problems. The 


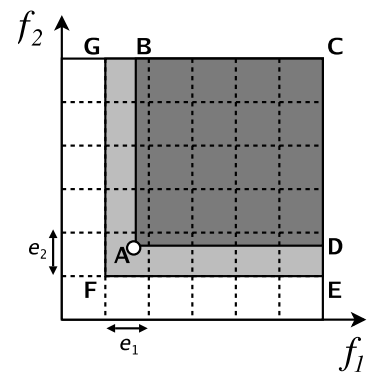

Figure 1: The 2-objective space is divided into hyper-boxes of size $\epsilon_{1} * \epsilon_{2}$ for objective $f_{1}$ and $f_{2}$ respectively. FGCE illustrates the space $\epsilon$-dominated by solution $A$ as compared to the actual dominated space ABCD. The $\epsilon$ parameters allow users to vary the precision of the boxes and hence the precision of the solutions obtained. Here, only one solution is allowed per hyper-box. Note: we are minimizing $f_{1}$ and $f_{2}$.

natural synergy between spatial evolutionary models and a multiobjective problem suggests that by varying the connectivity pattern of the network (that is, the local neighbourhood structure), selection pressure will also vary resulting in changes in the trajectory of the population. For multiobjective optimization problems with a large number of objectives, this heterogeneous spatial structure offers the possibility of improving the quality of solutions distributed across the Pareto-optimal front. In this study, we limit our analysis to the impact that alternative networks have on the algorithm performance for a number of scalable problems.

The remainder of the paper is organized as follows: In Section 2, we formally describe multiobjective optimization problems and the concept of $\epsilon$-dominance. In Section 3, we describe the complex network-based MOEA. The simulation experiments are described in Section 4 . This is followed by a discussion of the results in Section 5. We conclude the paper in Section 6 and outline directions for future research.

\section{BACKGROUND}

\subsection{Multiobjective optimization}

Multiobjective problems are problems that consist of a set of objective functions to be minimized or maximized subject to specified constraints. A multi-objective optimization problem can be stated generally as follows [4]:

$$
\begin{aligned}
\text { Minimize: } & f(x)=\left[f_{1}(x), f_{2}(x), \ldots, f_{k}(x)\right]^{T} \\
\text { subject to: } & g_{i}(x) \geq 0, i \in[1, \ldots, q] \\
& h_{i}(x)=0, i \in[1, \ldots, p]
\end{aligned}
$$

where $x$ is a vector of decision variables, $g_{i}$ is an inequality constraint, and $h_{i}$ is an equality constraint. A solution is said to dominate another if, for all objectives, it is as good as the other solution and better in at least one objective. That is, a solution $x^{*}$ dominates a solution $x$ (or $x^{*} \prec x$ ) iff

$\forall i f_{i}\left(x^{*}\right) \leq f_{i}(x) \wedge \exists j f_{j}\left(x^{*}\right)<f_{j}(x), i, j \in[1, \ldots, M]$

The set of non-dominated solutions with respect to all other feasible solutions form the Pareto-optimal set.

\section{$2.2 \epsilon$-dominance}

Laumanns et. al. [15], presented $\epsilon$-dominance as a method of ensuring both theoretical convergence (given infinite time) and a well-spread set of solutions along the Pareto-optimal front.

Given solution vectors $f$ and $g, f$ is said to $\epsilon$-dominate $g$ iff $\forall i \in 1, \ldots, M(1+\epsilon) . f_{i} \geq g_{i}$. Convergence can be guaranteed by maintaining an archive of $\epsilon$-dominating solutions and an update strategy such that solutions are never removed unless they are replaced by another dominating solution.

Through the use of the $\epsilon$ parameters (one for each objective) and identification vectors, the objective space is divided into hyper-boxes each of size $\epsilon_{j}$ (Figure 1). By restricting each hyper-box on the Pareto-optimal front to contain only one individual, a well-distributed and size-bounded set of solutions can be maintained.

Consider two solutions $\mathrm{A}$ and $\mathrm{B}$ with objective value pairs $\left(f_{1}, f_{2}\right)_{A}=(0.134,0.552)$ and $\left(f_{1}, f_{2}\right)_{B}=(0.132,0.554)$. If $\epsilon_{i}=\epsilon_{j}=0.01$ and $A$ already exists in the archive, B is not admitted to the archive since both belong to the same hyperbox $(0.13,0.55)$. Users are thus able to set the precision and number of the desired solutions by setting the $\epsilon$ parameter.

\section{COMPLEX NETWORK-BASED MOEA}

\subsection{A brief review of complex networks}

A network can be modelled as a graph $G(N, E)$ where $N$ is a finite set of nodes (vertices) and $E$ a finite set of edges (links) such that each edge is associated with a pair of nodes $i$ and $j$.

The degree $k_{i}$ of a node $i$ defines the total number of edges between node $i$ and all other nodes. The larger the degree, the "more important" the node is in a network. The node degree distribution function $P(k)$ is the probability that a randomly selected node has exactly $k$ edges. Here, we use $\left.<k_{i}\right\rangle$ to represent the mean degree. Other structural characteristics of interest are the clustering coefficient, $C$, which is the probability that two nearest neighbours of a node are also nearest neighbours of each other, and the characteristic path length, $L$, which measures the average separation between any two nodes in the network.

Four different network architectures were used in this study (see Fig. 2): (a) the regular network defined as a nearestneighbour coupled network (lattice) in which every node in the network is joined by a few of its neighbours; (b) the random network created by specifying that each pair of nodes is connected by an edge with uniform probability $p$; (c) the small-world network created by randomly re-wiring each edge with some probability $p<<1$; and (d) the scale-free network characterized by the distinctive connectivity distributions - the probability that a node selected uniformly at random has a certain number of links (degree) follows a power law governed by the relationship $P(k) \sim k^{-\gamma}$. The scale free networks were generated using the preferential attachment model in which we specified the initial number of nodes $(b)$ and the number of edges per node added $(e)$.

Space does not allow for a detailed description of the networks and their characteristics, however, a comprehensive overview can be found in [9]. 


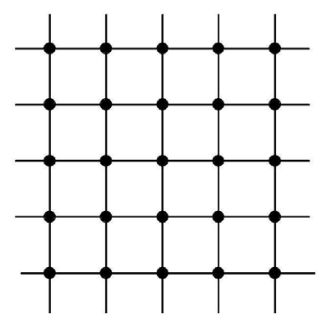

(a)

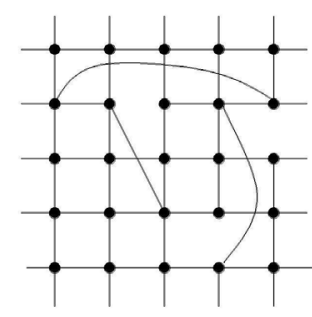

(b)

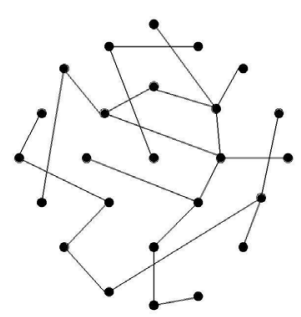

(c)

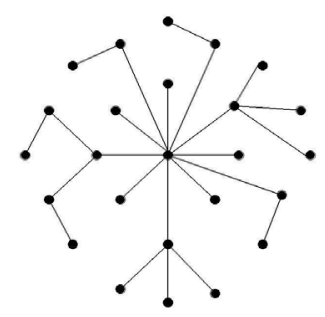

(d)

Figure 2: Complex network architectures. Here, we illustrate the structure of the alternative networks using 25 nodes for each. (a) Regular 2D lattice, (b) Small-world, (c) Random, and (d) Scale-free.

Algorithm 1: Complex network-based MOEA.

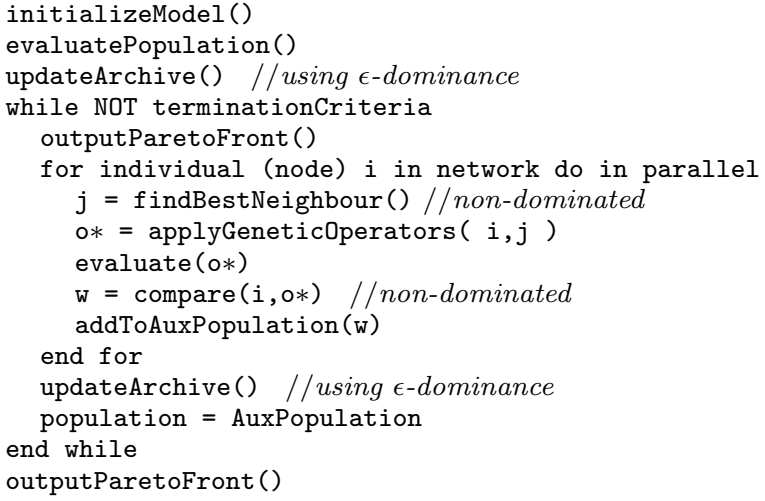

\subsection{Model details}

The complex network-based MOEA used in this study is an enhanced cellular evolutionary algorithm $[1,11,12]$. A key component of the model is the communication topology determined by the network architecture. Here, the individuals are mapped to the nodes of alternative complex networks and interact in their local neighbourhood. Algorithm 1 provides an overview of the key steps.

An important feature of the model is the variation in local neighbourhood size between networks - and within particular networks. Typically, the number of neighbours is not constant across the whole network. That is, the size of the local neighbourhood is determined by the degree $k_{i}$ of the current node $i$. This in turn means that the selection pressure will also vary. The exception to this rule is when a $2 \mathrm{D}$ regular lattice with Moore neighbourhood is used for which each individual has 8 neighbours.

In the selection phase, a relative non-dominance ranking mechanism is used to generate a pool of potential mates from the local neighbourhood. A crowding measure is then used to rank individuals in the mating pool. Here, the least crowed individual is viewed as better. This selection regime results in the identification of a "best" mate, $j$, for the current individual $i$. After the recombination stage, the resulting offspring are mutated. The parent occupying node $i$ and the resultant offspring are then compared using the dominance ranking mechanism. The nondominated individual is then copied into the auxilary population. In the event of a tie, one of the children or parent is selected randomly to enter the auxiliary population. After all nodes in the network have been processed, the auxiliary population is copied to the main population and the evolutionary cycle continues. An external archive is maintained using the $\epsilon$-dominance mechanism described in Section 2. The code for the archive was based on Deb, Mohan and Mishras' Epsilon-MOEA [5] archive implementation available at: http://www.iitk.ac.in/kangal/codes.shtml.

\section{SIMULATIONS AND RESULTS}

The goal of this study, was to characterize how the underlying network architecture impacts on solution quality as the number of objectives increases in scalable multiobjective optimization problems. Thus, a range of different networkproblem scenarios were examined.

\subsection{Implementation details}

Two benchmark scalable multiobjective problems were used: DTLZ4 and DTLZ5. A more comprehensive description of the test functions can be found in [8]. The actual implementation was adapted from the code available at http://www.tik.ee.ethz.ch/pisa/.

\section{DTLZ4}

Minimise:

$f_{1}(\mathbf{x})=\left(1+g\left(\mathbf{x}_{M}\right)\right) \prod_{j=1}^{M-1} \cos \left(x_{j}^{\alpha} \pi / 2\right)$

$f_{k}(\mathbf{x})=\left(1+g\left(\mathbf{x}_{M}\right)\right) \sin \left(x_{M-k+1}^{\alpha} \pi / 2\right) \prod_{j=1}^{M-k} \cos \left(x_{j}^{\alpha} \pi / 2\right)$

for $k=2,3, \ldots, M$,

where:

$g\left(\mathbf{x}_{M}\right)=\sum_{x_{i} \in \mathbf{x}_{M}}\left(x_{i}-0.5\right)^{2}=\sum_{j=M}^{P}\left(x_{j}-0.5\right)^{2}$

$0 \leq x_{i} \leq 1$, for $i=1,2, \ldots, P$,

\section{DTLZ5}

Minimise:

$f_{1}(\mathbf{x})=\left(1+g\left(\mathbf{x}_{M}\right)\right) \prod_{j=1}^{M-1} \cos \left(\theta_{j}\right)$

$f_{k}(\mathbf{x})=\left(1+g\left(\mathbf{x}_{M}\right)\right) \sin \left(\theta_{M-k+1}\right) \prod_{j=1}^{M-k} \cos \left(\theta_{j}\right)$,

for $k=2,3, \ldots, M$,

where:

$g\left(\mathbf{x}_{M}\right)=\sum_{x_{i} \in \mathbf{x}_{M}}\left(x_{i}-0.5\right)^{2}=\sum_{j=M}^{P}\left(x_{j}-0.5\right)^{2}$

$0 \leq x_{i} \leq 1$, for $i=1,2, \ldots, P$,

$\theta_{1}=x_{1} \pi / 2$

$\theta_{q}=\frac{\pi}{4\left(1+g\left(\mathbf{x}_{M}\right)\right)}\left(1+2 g\left(\mathbf{x}_{M}\right) x_{q}\right)$, for $q=2,3, \ldots,(M-1)$. 


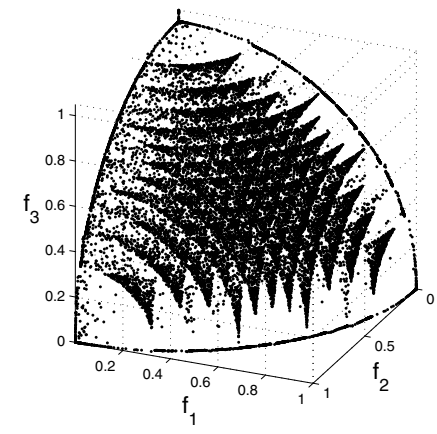

Figure 3: The non-dominated reference front for the 3 Objective DTLZ4 problem.

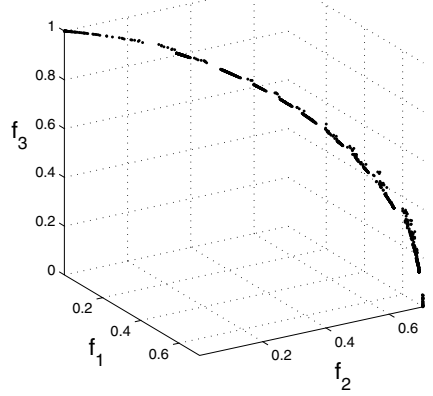

Figure 4: The non-dominated reference front for the 3 Objective DTLZ5 problem.

We set $\alpha=100$ in DTLZ4, and $P=M+9$ in both problems, where $M$ is the number of objectives. Real-encoding was used with SBX crossover. The mutation probability was set to $1 / P$, where $P$ is the number of decision variables. The selection regime was descibed in Section 3. The number of nodes in the network (population size) was set to 1024. For the external archive, values of $\epsilon=0.05$ were used for each scenario.

Table 1 lists the structural properties of each network used in this study.

Table 1: Network structural characteristics.

\begin{tabular}{llrrr}
\hline Network & Description & $\left\langle k_{i}\right\rangle$ & $\mathbf{L}$ & $\mathbf{C}$ \\
\hline $\mathbf{M}$ & $\begin{array}{l}\text { Regular 2D network } \\
\text { with } 8 \text { neighbourhoods. }\end{array}$ & 8.0 & 10.7 & 0.429 \\
SFA & $\begin{array}{l}\text { Scale-free, preferential } \\
\text { attachment, } b=10, e=2 .\end{array}$ & 4.0 & 4.5 & 0.013 \\
SFB & $\begin{array}{l}\text { Scale-free, preferential } \\
\text { attachment, } b=10, e=4 .\end{array}$ & 7.9 & 3.3 & 0.022 \\
SFC & $\begin{array}{l}\text { Scale-free, preferential } \\
\text { attachment, } b=10, e=6 .\end{array}$ & 11.9 & 2.9 & 0.036 \\
SFD & $\begin{array}{l}\text { Scale-free, preferential } \\
\text { attachment, } b=10, e=8 .\end{array}$ & 15.8 & 2.7 & 0.046 \\
SWA & $\begin{array}{l}\text { Small-world, base d=2, } \\
\text { r=1, } p=0.05\end{array}$ & 8.0 & 5.3 & 0.369 \\
SWB & $\begin{array}{l}\text { Small-world, base d=3, } \\
\text { r=1, } p=0.05\end{array}$ & 24.0 & 3.2 & 0.449 \\
SWC & $\begin{array}{l}\text { Small-world, base d=3, } \\
\text { r=2, } p=0.05\end{array}$ & 48.0 & 2.6 & 0.469 \\
R & $\begin{array}{l}\text { Erdös and Rényi random } \\
\text { graph with } p=0.05 .\end{array}$ & 50.7 & 2.0 & 0.049 \\
\hline
\end{tabular}

\subsection{Results}

For each combination of network, test problem, and number of objectives, the model was run for a maximum of 200 time steps. Thirty independent trials were completed for each scenario. In order to compare the performance of alternative network architectures, it is necessary to examine both the convergence time and spread of solutions across the Pareto-front. To do this comparison, we have constructed a reference set, $R$, by merging all of the archival non-dominated solutions found by each of the network-based models for a given problem across all Pareto-fronts (output every 10 generations) and trials. As an example, the reference sets for the 3-objective DTLZ4 and DTLZ5 problems are shown in Figures 3 and 4 respectively.

We have used the $I_{\epsilon}$ unary epsilon indicator [19] as the primary metric for comparison. Unfortunately, the hypervolume indicator, $I_{H}$, which measures the portion of the objective space that is weakly dominated by $R$, could not be used as the hypervolume is computational expensive when the number of objectives increases [17].

A non-parametric Kruskal-Wallis test was used to test for significant differences between scenarios. Tables 2 and 3 present a pair-wise statistical comparison between the complex network-based MOEAs using the $I_{\epsilon}$ indicator for DTLZ4 and DTLZ5 respectively as the number of objectives increased from two to six in steps of one. Here, we test the null hypothesis that the indicator value for the row entry is significantly better than the column entry ( $p$-value $<0.05)$. If the result for the given number of objectives is statistically significant, the number of objectives is listed in the cell.

Figures 5, 6 and 7 show the median convergence rate of $I_{\epsilon}$ for each of the network models on the DTLZ4 test problem for 2, 4 and 6 objectives repectively. Figures 8,9 and 10 show the median convergence rate of $I_{\epsilon}$ for each of the network

Table 2: Pair-wise statistical analysis of $I_{\epsilon}$ for the DTLZ4 problem.

\begin{tabular}{l|ccccccccc}
\hline & M & SFA & SFB & SFC & SFD & SWA & SWB SWC R \\
\hline M & - & $2-6$ & $3-6$ & $4-6$ & $4-6$ & & & 5,6 & 5,6 \\
SFA & & - & & & & & & & \\
SFB & & $2-4$ & - & & 4 & & & & \\
SFC & 3 & 2,3 & 3 & - & & 3 & & & \\
SFD & 3 & 2,3 & 3 & & - & 3 & & 5,6 & $4-6$ \\
SWA & & $3-6$ & $3-6$ & $4-6$ & $4-6$ & - & & 5,6 & $4-6$ \\
SWB & 3,6 & $2-6$ & $3-6$ & $3-6$ & $4-6$ & $2,3,6$ & - & -6 \\
SWC & 3 & $3-5$ & 3,4 & 3,4 & 3,4 & 3 & 3 & - & 4,6 \\
R & 3 & $2-4$ & 3 & 3 & 3,4 & 3 & 3 & & - \\
\hline
\end{tabular}

Table 3: Pair-wise statistical analysis of $I_{\epsilon}$ for the DTLZ5 problem.

\begin{tabular}{|c|c|c|c|c|c|c|c|c|c|}
\hline & $\mathbf{M}$ & SFA & SFB & SFC & SFD & SWA & SWI & SWC & $\mathbf{R}$ \\
\hline $\bar{M}$ & - & & & & & & & 3 & 3 \\
\hline SFA & & - & & & & & 3 & 3 & 3 \\
\hline SFB & 6 & 6 & - & & & 5,6 & 3 & 3 & 3 \\
\hline SFC & 4-6 & 4-6 & 4-6 & - & & 4-6 & 3 & 3 & 3 \\
\hline SFD & $3-6$ & 4-6 & 4-6 & 4-6 & - & 4-6 & 3 & 3 & 3 \\
\hline SWA & 2 & & 2 & 2 & & - & & 3 & 3 \\
\hline SWB & $2,4-6$ & $4-6$ & $2,4-6$ & $2,4-6$ & 5,6 & 4-6 & - & 3 & 3 \\
\hline SWC & $2,4-6$ & 4-6 & 4-6 & $2,4-6$ & 4-6 & 4-6 & $4-6$ & - & \\
\hline $\mathbf{R}$ & $2,4-6$ & 4-6 & $2,4-6$ & $2,4-6$ & 4-6 & 4-6 & 4-6 & & - \\
\hline
\end{tabular}


models on the DTLZ5 test problem for 2, 4 and 6 objectives repectively. The data points at time $t$ were found by calculating the $I_{\epsilon}$ values using the archive at time $t$ (across all trials) and the reference set $R$.

Figures 11,12,13 and Figures 14,15,16 plot the number of non-dominated solutions in the population at each iteration for all of the network models on problems DTLZ4 and DTLZ5 for 2, 4 and 6 objectives respectively.

\section{DISCUSSION}

As the number of objectives increases in a multiobjective optimization problem, many of the well-known algorithms (and variants thereof) struggle to find a range of solutions distributed across the Pareto-front. This is not really surprising as the Pareto-optimal front is a higher-dimensional surface, and it is difficult to identify solutions that are close in objective space. Obviously, the use of niching mechanisms, crowding procedures and $\epsilon$-dominance comparisons (to highlight the most common techniques) play a significant role in guiding the evolving population. However, the motivation behind this study, was to investigate how specific topological features of a network influence the dynamics of processes executed on the network.

An important advantage of spatially structured evolutionary algorithms, as compared to their standard panmictic counterpart, is their ability to constrain the diffusion rate of genetic material. The work of Giacobini et. al., [10] provides supporting evidence that small-world topologies allow for a tradeoff between robustness and speed of the search in single objective problems. In that study, they were able to show that the introduction of a limited amount of "randomness" built into a network was able to change the population dynamics. However, in our previous work [13], a random graph tended to produce the best performance for two-objective problems in terms of convergence speed and the dominance indicators when compared to small-world, scale-free and regular networks. Therefore, an important question addressed in this study was to determine whether this apparent trend was transferable to problems with more than two objectives.

The results of the pair-wise statistical tests listed in Tables 2 and 3 clearly indicate that there are significant difference in performance between networks. In general, as the number of objectives in a given problem increases, performance differences between network topologies are magnified.

The plots of $I_{\epsilon}$ convergence (Figures 5-10) suggest that the structural properties affect the convergence rate. In the case of the DTLZ4 problem, the relative ranking of network performance, in terms of the convergence of the $I_{\epsilon}$ indicator, tends to follow the value of the clustering coefficient. Here, higher $C$ values lead to improved performance. In contrast, for the DTLZ5 problem, the relative ranking of network performance is correlated with the value of $\left\langle k_{i}\right\rangle$. Higher values of $\left\langle k_{i}>\right.$ lead to better results.

Similar trends in both problems were evident between the regular lattice and small-world models with small re-wiring probabilities. As "shortcuts" were introduced (as a result of link re-wiring) the convergence rate improved. For larger re-wiring probabilities, the performance reflected that of the random networks. However, there were significant differences in convergence rates for the random network across the test problems considered. As the number of objectives increased for the DTLZ5 problem, there was a corresponding increase in the quality of results obtained. For the DTLZ4 problem, the performance of the random network dropped off as the number of objectives increased. The $I_{\epsilon}$ convergence rate for the scale-free networks were clearly slower for the DTLZ4 problem and were similar to networks with lower $<k_{i}>$ values for the DTLZ5 problem.

The time series plots of the number of non-dominated solutions found per generation (Figures 11-16) shed further light on the evolutionary dynamics. Initially, there were a relatively small number of non-dominated solutions in the population. As expected, for problems with more objectives, there are more non-dominated solutions in the population. There is often a decrease in the number of non-dominated solutions found (the magnitude of which is dependent on the number of objectives) in the early generations before niches are established and non-dominated solutions flourish.

For the DTLZ4 problem, the networks with higher $\left\langle k_{i}\right\rangle$ values tend to evolve a larger number of non-dominated solutions at each generation. For the DTLZ5 problem, networks with similar $\left\langle k_{i}\right\rangle$ display similar characteristics as the number of objectives increases. However, the shape of the time series plots for the DTLZ4 and DTLZ5 for particular networks are significantly different. The relative ordering of networks for the DTLZ4 problem is consistent across the number of objectives. Networks at the random end of the scale typically contain more non-dominated solutions per generation. In contrast, scale-free network consistently contain less non-dominated solutions per generation. As the number of objectives increased for the DTLZ5 problem, the networks with the larger $L$ values (regular lattice with Moore neighbourhood, small-world SWA) were able to find the greatest number of non-dominated solutions later in the run. Random networks tended to find a fixed number of non-dominated solutions, possibly indicating population convergence. Interestingly, the ability of scale-free networks to find non-dominated solutions improved as the number of objectives increased.

The locality of interactions plays an important role in the complex network-based MOEA. The selection pressure induced by the node degree and the corresponding local neighbourhood size have a direct affect on the rate of diffusion of information across the network. In 2D regular lattices and small-world models, the clustering coefficient is high. However, it appears that the average path length has a greater impact on the diffusion rate than the clustering coefficient. In networks with high mean node degrees, a few of the "best" non-dominated individuals will tend to be the parents of numerous offspring, leading to a homogenisation of the population due to offspring with similar chromosomes being produced. These networks will combine existing building blocks in the population quickly, but they do not excel in their capacity to find new building blocks. On the other hand, regular lattices (with Moore neighbourhood) and small-world networks with small re-wiring probability promote population heterogeneity. Novel building blocks can be discovered as genetic material gradually diffuses across the network. Here, good individuals require time to dominate the whole population.

Clearly, the network topology provides a mechanism for controlling the exploration/exploitation properties of the evolutionary algorithm. As the complexity (level of difficulty) of the problem increases, networks which limit the explorative powers of the algorithm - those networks with higher $<k_{i}>$ values - may not be as useful as networks that 


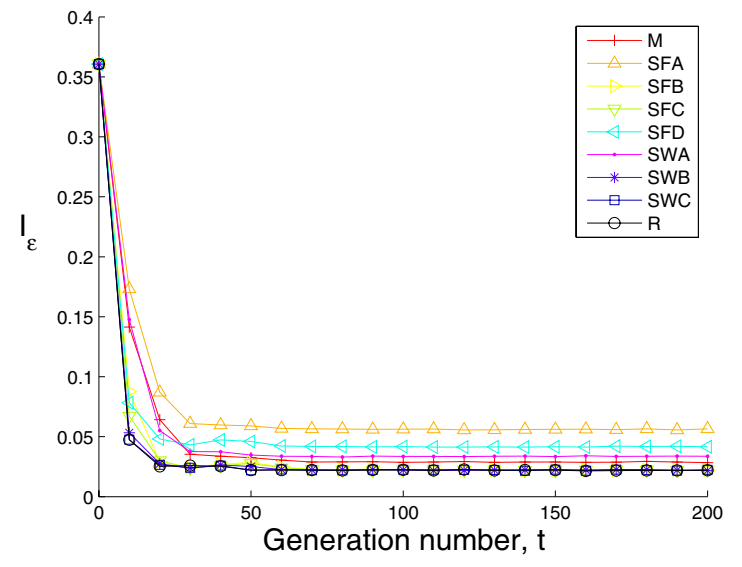

Figure 5: Convergence of $I_{\epsilon}$ for each network on the 2 objective DTLZ4 problem.

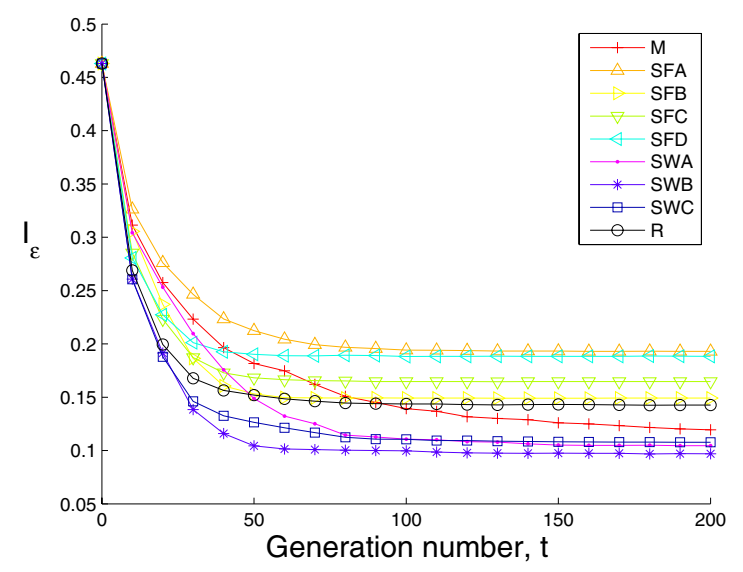

Figure 6: Convergence of $I_{\epsilon}$ for each network on the 4 objective DTLZ4 problem.

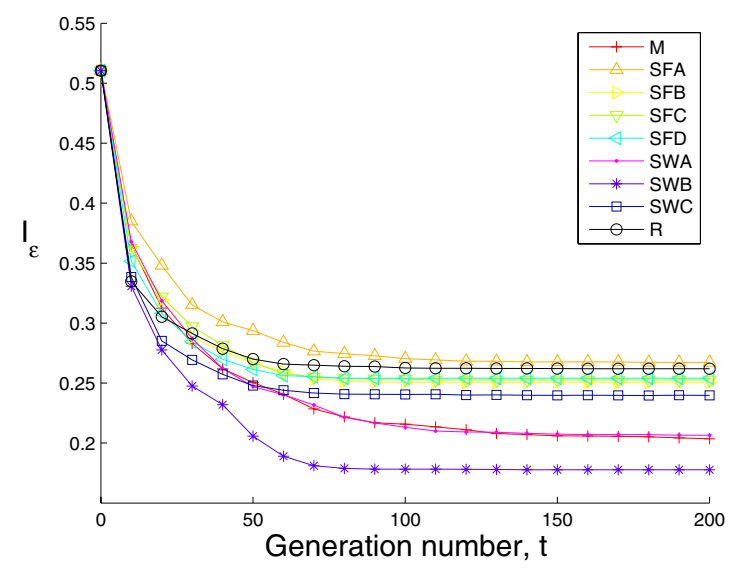

Figure 7: Convergence of $I_{\epsilon}$ for each network on the 6 objective DTLZ4 problem.

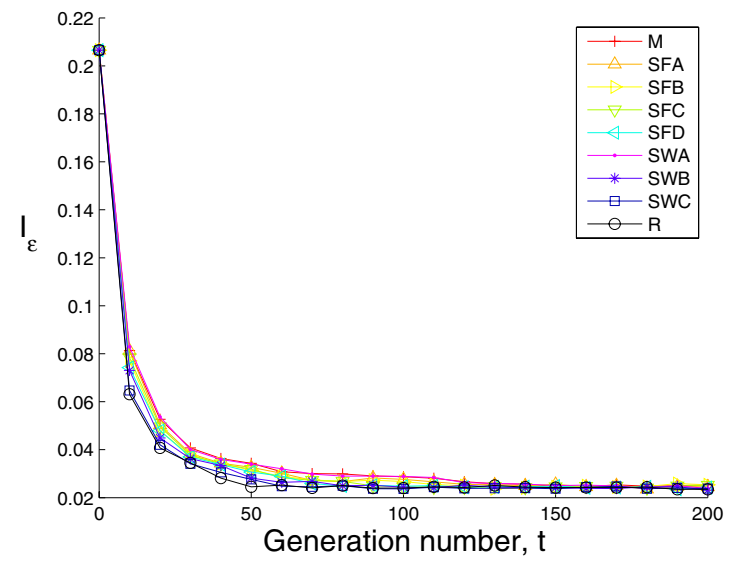

Figure 8: Convergence of $I_{\epsilon}$ for each network on the 2 objective DTLZ5 problem.

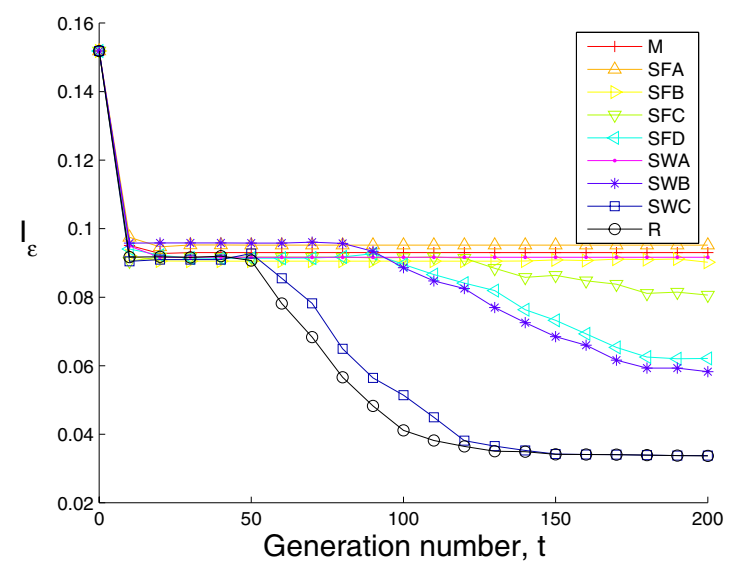

Figure 9: Convergence of $I_{\epsilon}$ for each network on the 4 objective DTLZ5 problem.

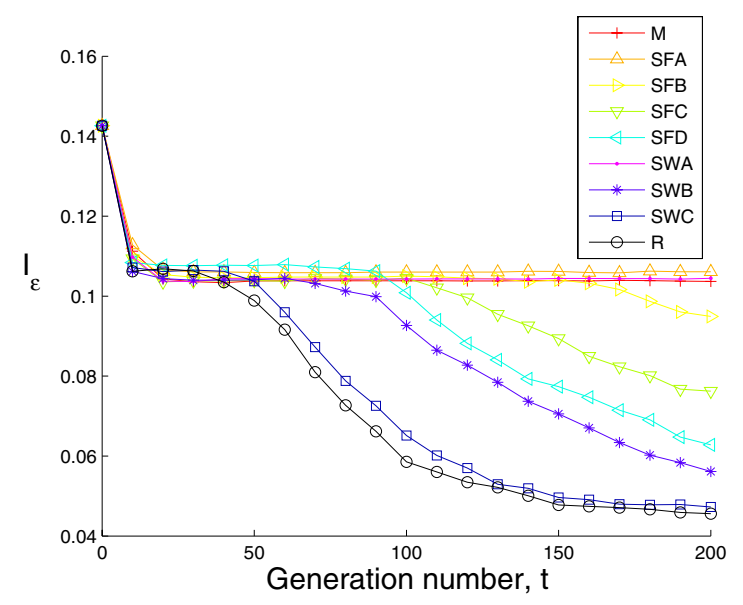

Figure 10: Convergence of $I_{\epsilon}$ for each network on the 6 objective DTLZ5 problem. 


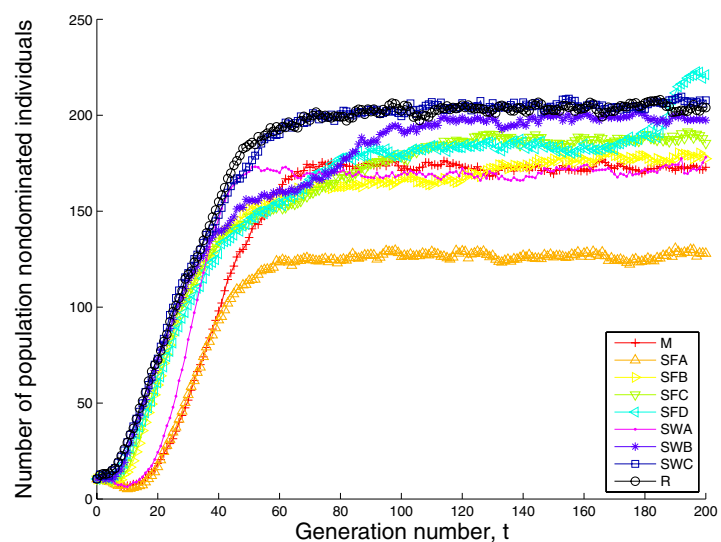

Figure 11: Number of population nondominated individuals over time for each complex network on the 2 objective DTLZ4 problem.

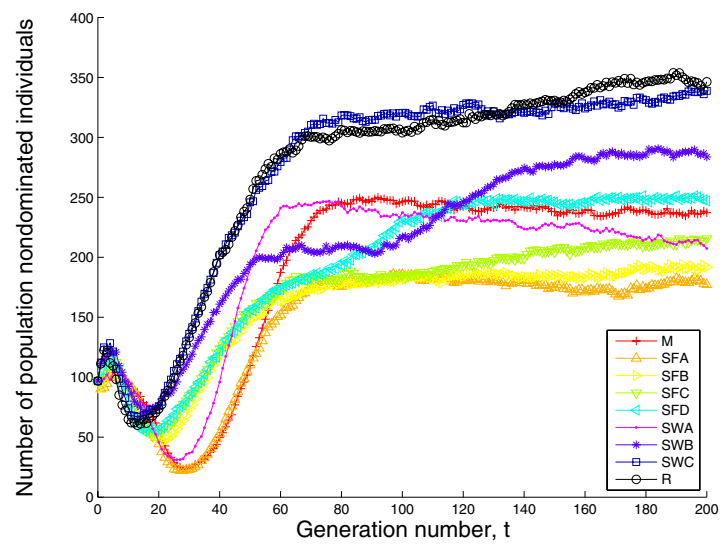

Figure 12: Number of population nondominated individuals over time for each complex network on the 4 objective DTLZ4 problem.

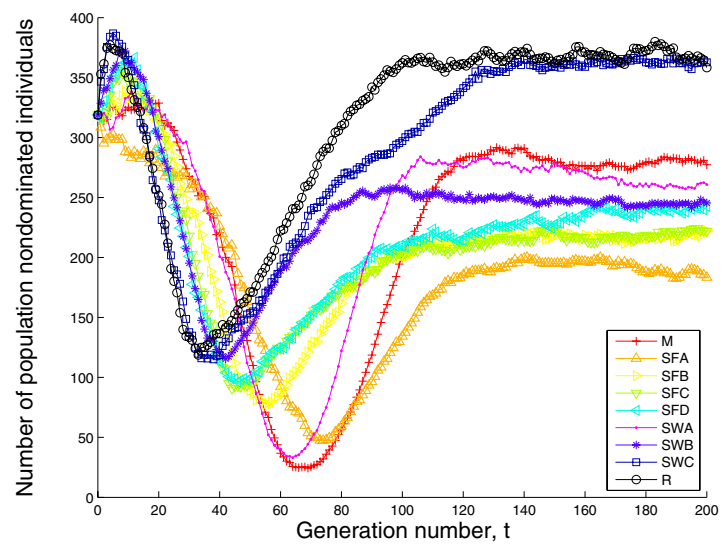

Figure 13: Number of population nondominated individuals over time for each complex network on the 6 objective DTLZ4 problem.

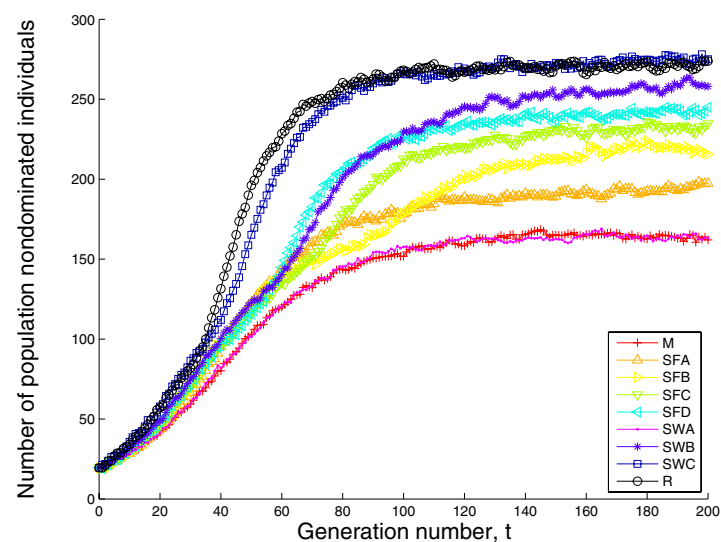

Figure 14: Number of population nondominated individuals over time for each complex network on the 2 objective DTLZ5 problem.

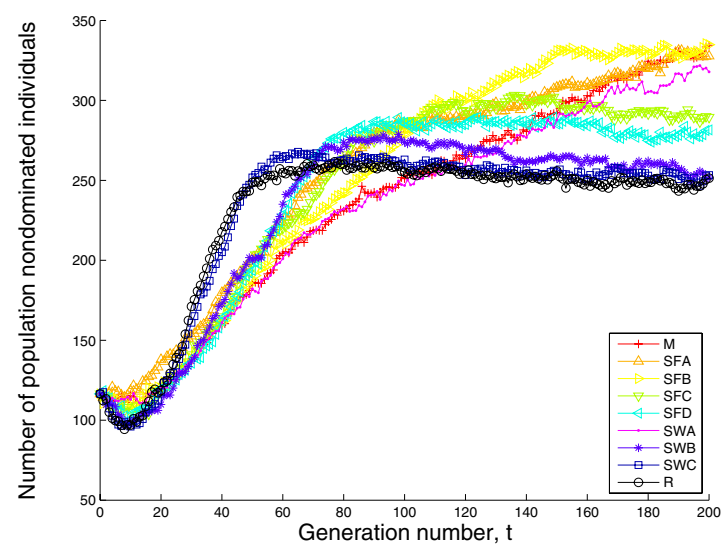

Figure 15: Number of population nondominated individuals over time for each complex network on the 4 objective DTLZ5 problem.

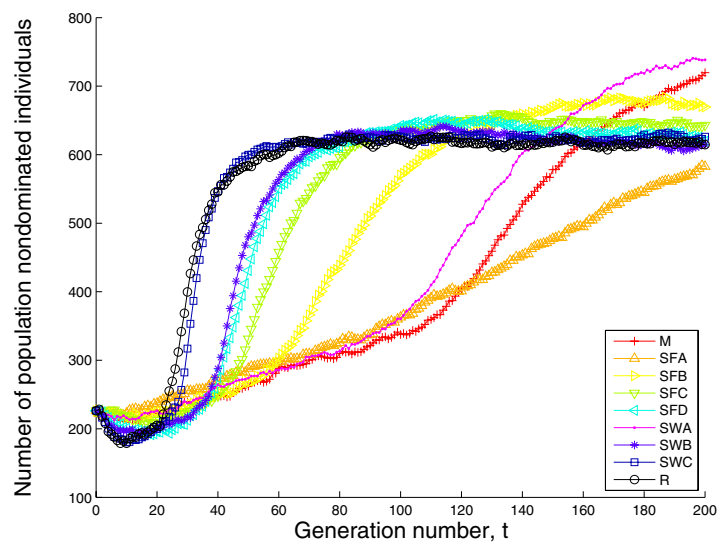

Figure 16: Number of population nondominated individuals over time for each complex network on the 6 objective DTLZ5 problem. 
constrain the rate of diffusion. For relatively small (simple problems), a random or scale free network (where exploitation can happen quickly due to high node connectivity) may perform better than other topologies. In contrast, a regular lattice or small-world network with limited re-wiring, may be a more appropriate choice for harder problems. However, even with sufficient time, the regular lattice may not be the superior performer (in terms of solution quality) since the diffusion process followed may not have allowed the best evolutionary trajectory to have been taken.

\section{CONCLUSION}

In this study, we have investigated how the underlying communication topology (spatial structure) of a Pareto-based evolutionary algorithm impacts on the performance of the algorithm using scalable multiobjective optimization problems. The simulation results showed that there were significant performance differences between networks as the number of objectives increased. As the complexity of the problem increases, the ideal network should change from one with a high mean degree distribution to a network with high clustering coefficient. In all cases, the introduction of a limited number of long-range connections (via link re-wiring) maintains population diversity and helps to promote solutions distributed along the Pareto-optimal front.

An interesting avenue for future work, would be to investigate what impact dynamic topologies might have on the evolutionary trajectories. Such networks could reconfigure during the evolutionary process to balance exploration and exploitation and adapt to a given problem.

\section{Acknowledgments}

This work was supported by an Australian Research Council Discovery Grant (DP0664674).

\section{REFERENCES}

[1] E. Alba and B. Dorronsoro. The exploration/exploitation tradeoff in dynamic cellular genetic algorithms. IEEE Transactions on Evolutionary Computation, 9:126-142, 2005.

[2] K. M. Bryden, D. Ashlock, S. Corns, and S. Wilson. Graph-based evolutionary algorithms. IEEE Transactions on Evolutionary Computation, 10:550-567, 2006.

[3] C. C. Coello, D. V. Veldhuizen, and G. Lamont. EA for Solving Multi-Objective Problems. Kluwer, 2002.

[4] K. Deb. Multi-Objective Optimization Using Evolutionary Algorithms. John Wiley and Sons, Ltd., Chichester, England, 2001.

[5] K. Deb, M. Mohan, and S. Mishra. A fast multi-objective evolutionary algorithm for finding well-spread pareto-optimal solutions. Technical Report 2003002, Kanpur Genetic Algorithms Laboratory, 2003.

[6] K. Deb, A. Pratap, S. Agarwal, and T. Meyarivan. A fast and elitist multiobjective genetic algorithm:
NSGA-II. IEEE Transactions on Evolutionary Computation, 6:182-197, 2002.

[7] K. Deb and J. Sundra. Reference point based multi-objective optimization using evolutionary algorithms. In M. Keijzer, editor, Proceedings of the Genetic and Evolutionary Computation Conference (GECCO-2006), ACM Press, pages 635-642. 2006.

[8] K. Deb, L. Thiele, M. Laumanns, and E. Zitzler. Scalable multiobjective optimization test problems. In Congress on Evolutionary Computation (CEC'2002), volume 1, pages 825-830. 2002.

[9] S. Dorogovtsev and J. Mendes. Evolution of Networks: From Biological Nets to the Internet and $W W W$. Oxford University Press, Oxford, 2003.

[10] M. Giacobini, M. Tomassini, and A. Tettamanzi. Takeover time curves in random and small-world structured populations. In Proceedings of the Genetic and Evolutionary Computation Conference (GECCO'05), pages 1133-1340, 2005.

[11] M. Giacobini, M. Tomassini, A. Tettamanzi, and E. Alba. Selection intensity in cellular evolutionary algorithms for regular lattices. IEEE Transactions on Evolutionary Computation, 9:489-505, 2005.

[12] M. Kirley. A cellular genetic algorithm with disturbances: Optimization using dynamic spatial interactions. Journal of Heuristics, 8:321-342, 2002.

[13] M. Kirley and R. Stewart. Multiobjective evolutionary algorithms on complex networks. In Fourth International Conference on Evolutionary Multi-Criterion Optimization. Lecture Notes Computer Science 4403, pages 81-95, 2007.

[14] J. D. Knowles and D. Corne. Approximating the nondominated front using the pareto archived evolution strategy. Evolutionary Computation, 8(2):149-172, 2000.

[15] M. Laumanns, L. Thiele, K. Deb, and E. Zitzler. Combining convergence and diversity in evolutionary multiobjective optimization. Evolutionary Computation, 10(3):263-282, 2002.

[16] D. Watts and S. Stogatz. Collective dynamics of "small-world" networks. Nature, 393:440-441, 1998.

[17] L. While. A new analysis of the lebmeasure algorithm for calculating hypervolume. In Evolutionary Multi-Criterion Optimization: Third International Conference, EMO 2005., Lecture Notes in Computer Science 3410, pages 326-340, 2005.

[18] E. Zitzler, M. Laumanns, and L. Thiele. SPEA2: improving the strength pareto evolutionary algorithm. Technical Report 103, Computer Engineering and Networks Laboratory (TIK), Swiss Federal Institute of Technology (ETH) Zurich, Gloriastrasse 35, CH-8092 Zurich, Switzerland, 2001.

[19] E. Zitzler and L. Thiele. Multiobjective evolutionary algorithms: A comparative case study and the strength pareto approach. IEEE Transactions on Evolutionary Computation, 3(4):257-271, Nov. 1999. 